\title{
Ajustes Conversacionales para el Aprendizaje de Contenidos en L2
}

Berta de Dios Garcia, Universitat Autònoma de Barcelona, España

\section{Introducción}

La secuencia didáctica (SD) en la cual debe contextualizarse la presente investigación lleva por título "Let’s musicalize in English!” (cf. Anexo A) y se llevó a cabo en un aula de tercero de ESO durante el tercer trimestre del curso 2008-09 en un instituto de Ripollet (Barcelona). Se trata de un proyecto piloto de Aprendizaje Integrado de Contenidos y Lenguas Extranjeras (AICLE) para enseñar una SD de música en inglés que fue diseñado por la profesora de música y la profesora de inglés, que es, además, la investigadora del presente trabajo.

Las aulas AICLE son un entorno ideal para estudiar fenómenos conversacionales porque en ellas, la lengua se usa para aprender a la vez que para comunicarse, la materia que se estudia es la que determina el tipo de lenguaje que se necesita aprender y la fluidez es más importante que la exactitud en el uso de la lengua. De acuerdo con Coyle (1999), una lección de AICLE bien planteada combina los siguientes elementos: 
Contenido - fomenta el progreso en el conocimiento, las destrezas y la comprensión de los temas específicos de un currículo determinado.

Comunicación - se usa la lengua para aprender mientras se aprende a usar la lengua misma.

Cognición - permite desarrollar las destrezas cognitivas que enlazan la formación de conceptos, conocimientos y lengua.

Cultura - facilita la exposición a perspectivas variadas y a conocimientos compartidos que nos hagan más conscientes del otro y de uno mismo.

La SD que utilizamos trataba sobre los musicales del siglo XX y estaba dividida en dos tipos de sesiones: sesiones de expertos y sesiones de todo el grupo. Las sesiones de experto constaban de dos partes. En la primera, toda la clase se dividía en tres grupos y cada grupo tenía que leer y colaborar en la comprensión de tres textos diferentes relacionados temáticamente. Cada integrante del grupo tomaba apuntes de los contenidos más relevantes de su texto. En la segunda parte, cada cual se reunía con dos personas de los otros dos grupos junto a los cuales formaba un trío de expertos, cada cual en su texto correspondiente. Cada estudiante enseñaba, a partir del resumen que había realizado, lo que había aprendido en su texto a los otros dos, que tomaban los respectivos apuntes. Estos tríos de alumnado experto se mantuvieron uniformes durante toda la SD. 
Todo el proceso lo hacía el alumnado de forma autónoma y la profesora sólo supervisaba las conversaciones a distancia.

Las sesiones de todo el grupo consistían en audiciones musicales relacionadas con los contenidos de la sesión de expertos del día anterior. Al final de la SD hubo un examen sobre los contenidos trabajados en todas las sesiones. El alumnado sabía que habría un examen al final de la SD, para el cual debían prepararse mediante los apuntes tomados, pero no sabía que lo harían colaborativamente entre cada trío de expertos.

\section{Objectivos}

En el presente trabajo pretendemos analizar las interacciones en las conversaciones entre tres alumnos en un aula AICLE para comprobar qué características en dichas interacciones pueden favorecer la construcción de conocimientos de contenidos no lingüísticos. Para ello, centraremos nuestro estudio en el análisis de la negociación de la intersubjetividad y, concretamente, en el análisis de las reparaciones y compararemos las características que encontremos con las características que se han asociado a las conversaciones ordinarias. 


\section{Marco teórico}

Por lo que respecta al marco teórico, debemos empezar por aclarar cómo entendemos algunos conceptos que vamos a usar. En primer lugar, entendemos que el aprendizaje se produce mediante la co-construcción, por parte de los participantes en las interacciones, de los significados del mensaje a medida que la interacción se desarrolla (modelo de comunicación constructivista). Por ello, creemos que el mejor método (cf. Metodología) para analizar el aprendizaje es el Análisis de la Conversación (AC) porque permite “emular el carácter emergente que tiene una interacción para los participantes” y examinar cómo "las acciones y conductas individuales son interpretadas y mutuamente ratificadas por los interlocutores" (Dalton-Puffer 2007:37).

Por lo tanto, hay que definir algunos conceptos propios del AC. El AC nos informa sobre la organización de la secuencia de turnos en la conversación ordinaria (Seedhouse 2005:168). Un turno es una serie de habla construida colaborativamente por los interlocutores que está constituida por una o varias unidades de construcción de turnos (TCU en inglés). Una TCU puede ser una oración, un sintagma o una palabra y permite a los interlocutores construir de forma progresiva y colaborativa turnos sintagmáticamente más complejos (Markee 2000:84) sin las exigencias de tener que 
producir individualmente oraciones enteras (Markee 2000:86). La organización de la secuencia de turnos está basada en dichas TCU y en los lugares de relevancia transitiva (TRP en inglés). Cuando aparece un TRP se ponen en juego una serie de normas relacionadas con la transición de los hablantes, los cuales pueden escoger hablar o ceder el turno, lo cual depende en gran medida del objetivo institucional de la interacción (Seedhouse 2005:168).

En la presente investigación se analizarán conversaciones entre el alumnado sin la presencia de la profesora. En estos casos, la tendencia es encontrar un sistema de interacciones controladas en igualdad de poder, lo que nos permite establecer ciertos paralelismos con las conversaciones ordinarias. Compararemos pues los datos recogidos con las características teóricas de las conversaciones ordinarias (Markee 2000:97). Según Markee, cuanto más local es el control de las interacciones, más probable es que todos los interlocutores sean considerados como hablantes lícitos, los cuales tienden a hablar secuencialmente sin solapamientos. Sin embargo, la distribución de turnos entre los interlocutores suele ser desequilibrada. En este tipo de interlocuciones, el tamaño de cada TCU suele ser reducido (sintagmas o palabras) y es la combinación de varios TCU de diferentes hablantes lo que incrementa la complejidad sintáctica del turno. Contrariamente, hay características que 
alejan las interacciones que analizaremos de las conversaciones ordinarias: la duración está marcada por el horario escolar y el contenido viene determinado por lo que la profesora había preparado anticipadamente.

Otro aspecto relevante a tener en cuenta es que las conversaciones analizadas son en lengua extranjera (L2), es decir, entre hablantes no nativos. Varios estudios han investigado las características específicas de estas interacciones y han concluido que presentan una elevada frecuencia de negociaciones de significado, probablemente por la falta de un bagaje compartido, incluso en casos como éste, en que los hablantes comparten la misma lengua materna (L1), porque la L2 es ajena para todos (Varonis y Gass 1985; citado en Markee, 2000:104). Esta construcción de los significados de los mensajes como resultado de un esfuerzo social compartido (intersubjetividad) se puede considerar un ejemplo de cognición socialmente distribuida que nos remite de nuevo al modelo de comunicación constructivista. La intersubjetividad no es un estado estático e imperturbable, es decir, podemos esperar que los hablantes interactúen aportando contenidos para su mantenimiento (Dalton-Puffer 2007:68). Sin embargo, al tratarse de una conversación entre aprendices, éstos tienden a concebir la negociación de significados como una "solución local a un problema local” (Markee 2000:123). Los 
interlocutores no considerarán las relaciones semánticas observables como oportunidades de aprendizaje, mas intentarán hallar la forma más sencilla para reparar posibles escollos en la construcción de la intersubjetividad para salir del paso lo antes posible. En este caso, en que la lengua de interacción es la L2, como veremos, la forma más sencilla de reparación será el cambio de código a L1 de los elementos de la TCU que planteen problemas.

La reparación es el recurso más frecuente para mantener la intersubjetividad. El AC define la reparación como el tratamiento de un problema que surge en la interacción, entendiendo como problema cualquier cosa que los participantes consideren que impide la comunicación y es reparable (Seedhouse 2005:168). Desde la perspectiva del AC, todas las reparaciones suelen estar indicadas per alguna marca de inicio de reparación (pausas, alargamientos de sonido, encabalgamientos, muletillas lingüísticas, etc.). Hay que distinguir diferentes tipos de reparación en función de cuatro parámetros: (a) su posición en relación con el problema, (b) quién la inicia (el mismo hablante, autoinicio, u otro interlocutor, heteroinicio), (c) quién la completa (el mismo hablante, autocompleción, u otro interlocutor, heterocompleción) y (d) si el esfuerzo de reparación tiene éxito o no (Markee 2000:102-103). En relación a estos cuatro 
parámetros, en la presente investigación pretendemos comprobar si los datos cumplen las siguientes características recopiladas por otros investigadores (cf. Figura 1).

\begin{tabular}{|l|l|l|}
\hline $\begin{array}{l}\text { Contexto } \\
\text { donde se da la } \\
\text { característica }\end{array}$ & Característica & $\begin{array}{l}\text { Autor/a de la } \\
\text { investigación }\end{array}$ \\
\hline $\begin{array}{l}\text { Conversaciones } \\
\text { ordinarias en en } \\
\text { L2. }\end{array}$ & $\begin{array}{l}\text { Las reparaciones tienden a ser } \\
\text { autocompletadas. }\end{array}$ & $\begin{array}{l}\text { Schwartz, 1980; } \\
\text { citado en Markee } \\
2000: 110 .\end{array}$ \\
\hline
\end{tabular}

\begin{tabular}{|l|l|l|}
\hline $\begin{array}{l}\text { Conversaciones } \\
\text { en L2 en clases } \\
\text { centradas en la } \\
\text { lengua. }\end{array}$ & $\begin{array}{l}\text { Las reparaciones más } \\
\text { frecuentes se encuentran en la } \\
2^{\mathrm{a}^{1}} \text { y } 3^{\mathrm{a} 2} \text { posición respecto al } \\
\text { problema. }\end{array}$ & Markee 2000:113. \\
\hline
\end{tabular}

Fig. 1. Características de las reparaciones que pretendemos comprobar en nuestros datos

Centrar la investigación en el estudio de las reparaciones nos parece pertinente, dado que nos permite ver cómo se alinean los discursos de los interlocutores e identificar las inferencias que aporta el alumnado en el mismo momento en que surgen. ¿Cómo podemos comprobar que las aserciones de mutua comprensión del alumnado (intersubjetividad) son dignas de confianza (Markee 2000:136)? Comprobando que dicha intersubjetividad dio sus frutos (al menos a corto plazo) en el examen. En otras palabras, constatando que la intersubjetividad en las conversaciones produjo construcción de conocimientos de contenidos no lingüísticos en el alumnado, el cual pudo responder satisfactoriamente a las preguntas del examen al final de la SD. 


\section{Recogida de datos}

Los datos que se utilizaron en la investigación proceden de dos fuentes diferentes. Por un lado, se realizaron grabaciones de todas las interacciones de todos los tríos de expertos (segunda parte de las sesiones de expertos). El alumnado sabía que su profesora los estaba grabando porque cada trío era responsable de su grabadora, pero no sabía para qué. Por otro lado, se tuvieron en cuenta las respuestas al examen del final de la SD.

Aunque se grabó a todos los tríos de expertos, el ruido presente en la mayoría de las grabaciones nos llevó a escoger las grabaciones del trío formado por Ir, Lu y Sa, porque eran las de mejor calidad.

Posteriormente, se leyeron las respuestas al examen hecho colaborativamente por dicho trío (cf. Anexo C) y se compararon con los contenidos de los textos leídos y explicados por ellos, donde se encontraba la información pertinente (cf. Anexo B). Se escogieron dos respuestas para dar cuenta de la diversidad de vínculos posibles entre el contenido de los textos originales y sus resultados en el examen: una respuesta en la cual el contenido no coincidía con el del texto correspondiente (respuesta a la pregunta 5) y una en la cual el contenido era satisfactoriamente parecido al del texto pertinente (pregunta 7). 
Posteriormente, se acudió a las grabaciones de las sesiones de expertos donde se habían tratado los contenidos seleccionados y los fragmentos relevantes se transcribieron de forma refinada. Nos habría gustado tener en cuenta también la grabación del día del examen, cuando Ir, Lu y Sa colaboraban para contestar a las preguntas, pero la mala calidad de la grabación también nos impidió tomar en consideración dichos datos.

\section{Análisis de los datos}

Para analizar en los datos recogidos cómo se encarnaba en la conversación la construcción de los conocimientos pertinentes seguimos las pautas de AC propuestas por Markee (2000:118119), según el cual, la metodología debe poder:

- Basarse en evidencias empíricas de la competencia interaccional de los interlocutores, consideradas desde un punto de vista émico.

- Basarse en colecciones de datos relevantes, extraídos de transcripciones completas de sucesos comunicativos, temáticamente relacionadas entre sí.

- Explotar el potencial analítico de transcripciones refinadas.

- Identificar éxitos y fracasos de aprendizaje, como mínimo a corto plazo.

- Demostrar cómo el significado se construye como fenómeno social.

Queremos cuestionar aquí la transferibilidad de los resultados obtenidos. Creemos que, al tratarse de un estudio de caso con un bajo grado de selectividad, no podemos asegurar que los 
resultados obtenidos sean generalizables, las observaciones que podamos hacer sólo son preliminares.

\section{Análisis}

Procederemos al análisis de los fragmentos en el orden en que tuvieron lugar. La simbología de Transcripción se encuentra referenciada en el anexo D.

Comenzamos con el fragmento 1 (Porgy and Bess), correspondiente a la sesión 2 (cf. Anexo B) y a la pregunta 5 del examen (cf. Anexo C). El literal del texto que Lu debía comunicar a Ir y Sa decía:

$<<$ Some critics consider this opera a racist description of African Americans. Several of the members of the original cast said that they thought that their characters were stereotypes: African Americans lived in poverty, took drugs and solved their problems fighting $>>$

Fig. 2. Texto original que Lu debía transmitir

Posteriormente, el trío contestó lo siguiente en el examen:

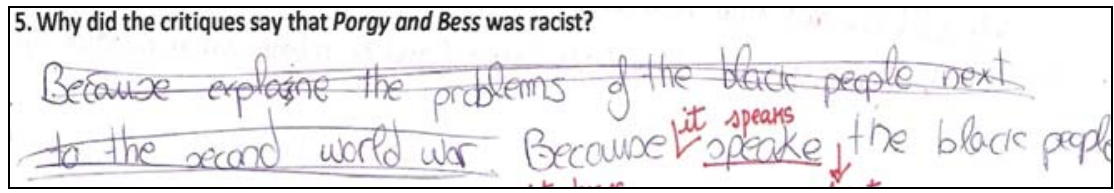

Fig. 3. Respuesta al examen correspondiente al contenido de la figura 2

La respuesta no es correcta, dado que Porgy and Bess no fue considerada racista por hablar de personas negras sino por presentar estereotipos de los afroamericanos. 
Ahora veamos cómo negoció el trío los significados relevantes en la conversación pertinente:

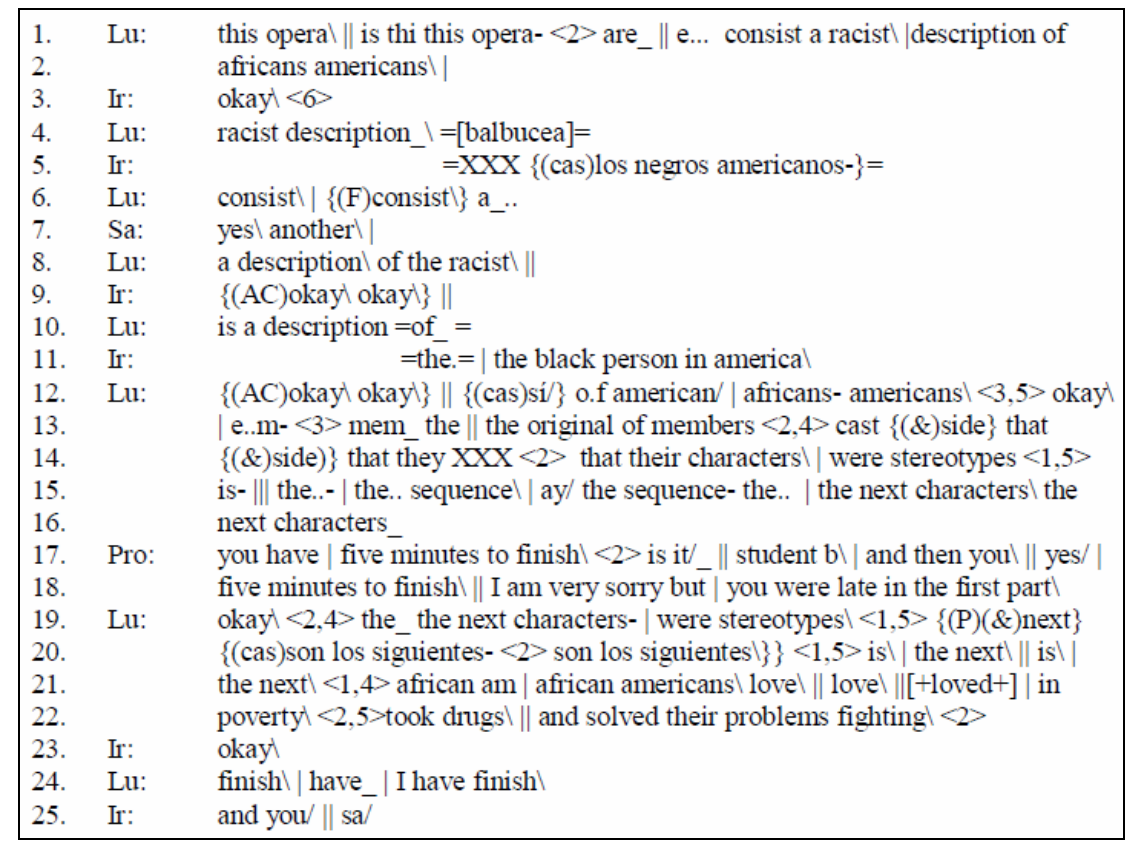

Fig. 4. Transcripción de la conversación en que trataban los contenidos de la figura 2

Nos encontrarnos aquí con un fragmento controlado de forma local en el que Lu, al ser el que debe exponer el texto, tiene las intervenciones más largas pero en el que Ir y Sa interrumpen en varias ocasiones su discurso la mayoría de ellas no para negociar significados sino para instarla a proseguir su discurso y se producen dos solapamientos (intervenciones 4 y 5, 10 y 11) porque los tres se consideran en igualdad de poder, como en una conversación ordinaria. 
Lu expone su primera idea en las intervenciones 1, 2, 4, 6, 8, 10 y 12. En la intervención 4 encontramos la primera evidencia de reparación. En este caso, se trata, por lo tanto, de una reparación autoiniciada en tercera posición respecto a lo que Lu considera el problema, el término “africans americans”. Los intentos de reparación corresponden a $\operatorname{Lu}(4,6,8,10$ y 12) y a Ir (5 y 11) pero no es hasta el final de la 12 que, finalmente, Lu acepta que el significado es compartido por los tres alumnos.

Por lo tanto, se trata de una reparación autocompletada. Vemos que en las dos ocasiones en que Ir colabora con Lu, ésta acaba la oración iniciada por aquél en el TCU anterior, una vez efectuando un cambio de código al castellano (5) y la otra manteniendo el código (11). Este cambio de código resulta la primera evidencia observable de lo establecido por Markee (2000:123): que el alumnado concibe la negociación de significados como una "solución local a un problema local”, sin orientar la conversación hacia objetivos de aprendizaje. En total, se requieren nueve intervenciones (4-12) entre el inicio de la reparación y su compleción. Ir y Sa, por otro lado, interrumpen el discurso de Lu en varias ocasiones (3, 7 y 9) para comunicar a Lu que comprenden lo que pretende decir. Por lo tanto, nos encontramos con el primer caso de evidencias observables de intersubjetividad. 
Entre el turno 13-16 y el 19-22, Lu expone su segunda idea. Esta vez, sin embargo, construye el mensaje solo, sin más interrupciones que la de la profesora (17-18), la cual no participa en la conversación, sino que interviene para gestionar el tiempo dedicado a la actividad. Sólo al final de la disertación solitaria de Lu encontramos una aserción de comprensión por parte de Ir (23), aunque no hay evidencias observables de que dicha comprensión sea digna de confianza. En la intervención 15 encontramos una reparación en primera posición respecto al problema identificado por Lu: “sequence”, que se completa en la misma intervención. Se trata, pues, de una reparación autoiniciada y autocompletada. Hay otra evidencia de una reparación equivalente en la intervención 19 de $\mathrm{Lu}$, donde el problema detectado por Lu es “next”. Para completarla, Lu efectúa un cambio de código al castellano, en voz baja, lo que vuelve a dejar constancia de una "solución local a un problema local”.

En general, vemos que las reparaciones de este fragmento tienen éxito y son iniciadas y completadas por el mismo interlocutor, por un motivo semántico propiciado por el uso de L2 y están completadas sin ánimo de evolucionar en el aprendizaje sino para salir del paso rápidamente en la construcción de la intersubjetividad. 


\begin{tabular}{|c|c|c|c|c|c|c|c|}
\hline 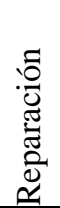 & 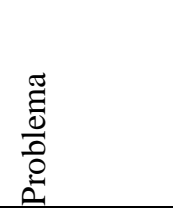 & 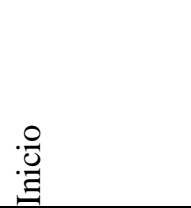 & 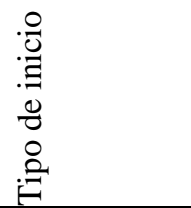 & 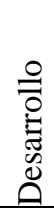 & $\underset{\substack{x \\
.1}}{.0}$ & 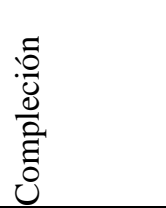 & 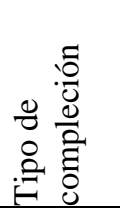 \\
\hline 1 & $\begin{array}{l}\text { Lu: } \\
\text { "africans } \\
\text { americans" } \\
\text { (2) }\end{array}$ & $\begin{array}{l}\text { Lu: } \\
\text { “[balbucea]” } \\
\text { (4) }\end{array}$ & $\begin{array}{l}3^{\mathrm{a}} \text { posición } \\
\text { autoiniciada }\end{array}$ & $\begin{array}{l}4- \\
12\end{array}$ & Sí & 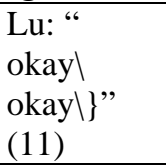 & $\begin{array}{l}\text { Auto- } \\
\text { Comple } \\
\text {-tada }\end{array}$ \\
\hline 2 & $\begin{array}{l}\text { Lu: } \\
\text { "sequence" } \\
\text { (15) }\end{array}$ & $\begin{array}{l}\text { Lu: " ay/ the } \\
\text { sequence" } \\
\text { (15) }\end{array}$ & $\begin{array}{l}1^{\mathrm{a}} \text { posición } \\
\text { autoiniciada }\end{array}$ & - & Sí & $\begin{array}{l}\text { Lu: "the } \\
\text { next } \\
\text { characters" } \\
\text { (15) }\end{array}$ & $\begin{array}{l}\text { Auto- } \\
\text { comple } \\
\text {-tada }\end{array}$ \\
\hline 3 & $\begin{array}{l}\text { Lu: “next” } \\
\text { (19) }\end{array}$ & $\begin{array}{l}\text { Lu: "son los } \\
\text { siguientes" } \\
\text { (20) }\end{array}$ & $\begin{array}{l}1^{\text {a }} \text { posición } \\
\text { autoiniciada }\end{array}$ & - & Sí & $\begin{array}{l}\text { Lu: “ son } \\
\text { los } \\
\text { siguientes” } \\
\text { (20) }\end{array}$ & $\begin{array}{l}\text { Auto- } \\
\text { comple } \\
\text {-tada }\end{array}$ \\
\hline
\end{tabular}

Fig. 5. Reparaciones del fragmento 1

\section{Fragmento 2: West Side Story}

En segundo lugar, analizaremos el fragmento correspondiente a la sesión 4 (cf. Anexo B) y a la pregunta 7 del examen (cf. Anexo C). En este caso, fue Ir la experta encargada de enseñar los siguientes contenidos:

$<<$ West Side Story is based on William Shakespeare's Romeo and Juliet but it is set in the West Side neighbourhood of New York in the 1950s. It explains the rivalry between two teenage gangs of different cultures: the Jets (white Americans) and the Sharks (latin Puerto Rican immigrants). The young protagonist, Tony, who belongs to the Jets, falls in love with Maria, the sister of Bernardo, the Shark's leader. The couple defies the traditional rivalry between the Jets and the Sharks, whose members don't understand their love. $\gg$

Fig. 6. Texto original que Ir debía transmitir

En el examen, el grupo contestó: 


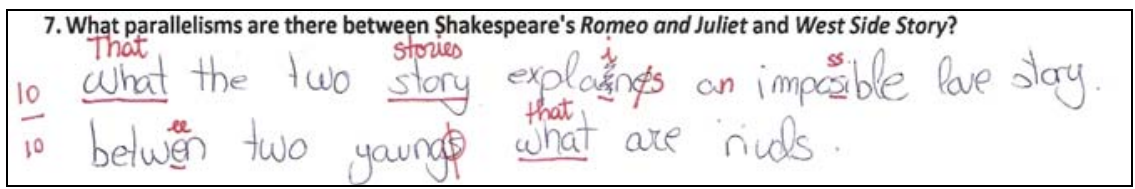

Fig. 7. Respuesta al examen correspondiente al contenido de la figura 6

En este caso, observamos que el resumen del trío sintetiza correctamente lo establecido en el texto original. Ahora veamos cómo se encarnó en la conversación la construcción de esta contenido: 


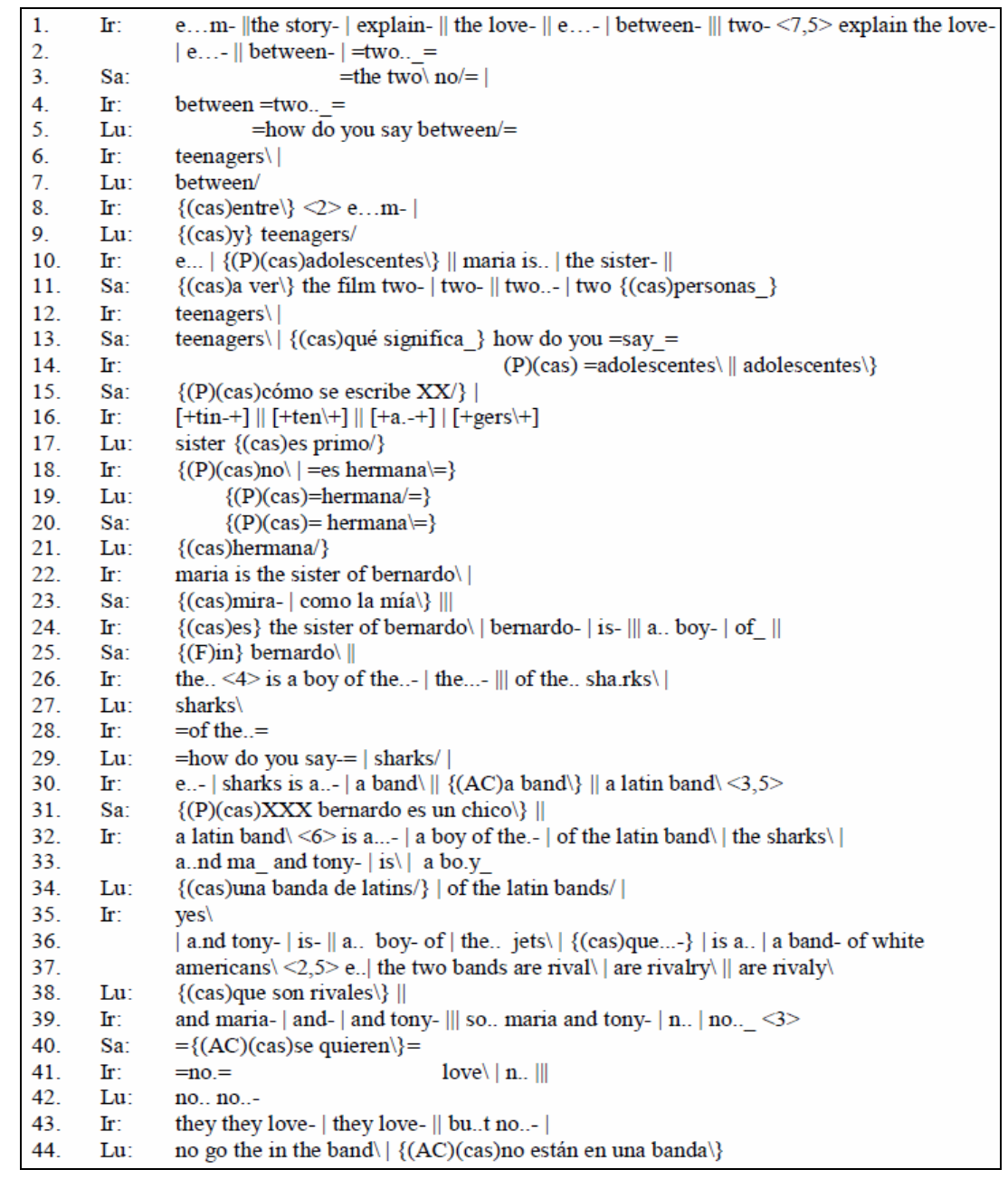

Fig. 8. Transcripción de la conversación en que trataban los contenidos de la figura 6

En este fragmento se tratan en total seis ideas: "la historia explica el amor entre dos adolescentes" (1-10, 11-16), "Maria es hermana de Bernardo" (10, 17-24, 25), "Bernardo pertenece a los Sharks, que es una banda latina” (24, 26-32, 34-35), “Tony 
pertenece a los Jets, que es una banda de blancos americanos” (33, 36-37), "las dos bandas son rivales" (37-38) y "Maria y Tony no van con las bandas” (39-44). En este caso vemos que Ir, la experta en dichos contenidos, hace unas intervenciones más cortas que $\mathrm{Lu}$ en el fragmento 1 , porque $\mathrm{Sa}$ y $\mathrm{Lu}$ la interrumpen continuamente. Es, por tanto, una clara evidencia de una conversación controlada de forma local en igualdad de poder.

En este corto fragmento nos encontramos con ocho reparaciones muy diferentes. Para facilitar su análisis, hemos hecho una tabla que detalla los diferentes parámetros que analizamos (cf. Figura 9):

\begin{tabular}{|c|c|c|c|c|c|c|c|}
\hline 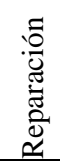 & 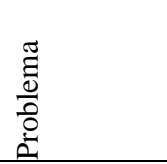 & 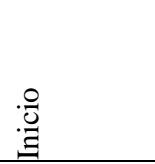 & 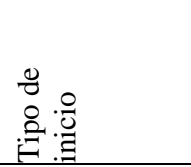 & 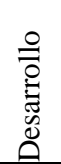 & : & 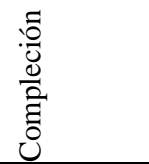 & 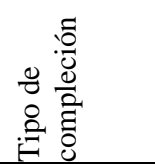 \\
\hline 1 & $\begin{array}{l}\text { Ir: "two" } \\
\text { (1) }\end{array}$ & $\begin{array}{ll}\text { Sa: "the } \\
\text { two } \\
\text { (3) no/" }\end{array}$ & $\begin{array}{l}2^{\mathrm{a}} \text { posición } \\
\text { heteroiniciada }\end{array}$ & - & No & - & - \\
\hline 2 & $\begin{array}{l}\text { Ir: } \\
\text { "between" } \\
\text { (4) }\end{array}$ & $\begin{array}{l}\text { Lu: how } \\
\text { do you say } \\
\text { between/ } \\
\text { (5) }\end{array}$ & $\begin{array}{l}2^{\mathrm{a}} \text { posición } \\
\text { heteroiniciada }\end{array}$ & $5-8$ & Sí & $\begin{array}{l}\text { Ir: } \\
\text { “entrel” } \\
\text { (8) }\end{array}$ & $\begin{array}{l}\text { Auto- } \\
\text { completada }\end{array}$ \\
\hline 3 & $\begin{array}{l}\text { Ir: } \\
\text { "teenagers" } \\
\text { (6) }\end{array}$ & $\begin{array}{l}\text { Lu: “y } \\
\text { teenagers/” } \\
\text { (9) }\end{array}$ & $\begin{array}{l}4^{\text {a }} \text { posición } \\
\text { heteroiniciada }\end{array}$ & $\begin{array}{l}- \\
16\end{array}$ & Sí & $\begin{array}{l}\text { Ir: “[+tin- } \\
+] \\
{[+ \text { ten } \backslash+] \|} \\
{[+ \text { a.-+] }} \\
{[+ \text { gers } \backslash+]} \\
(16)\end{array}$ & $\begin{array}{l}\text { Auto- } \\
\text { completada }\end{array}$ \\
\hline 4 & $\begin{array}{l}\text { Ir: "sister" } \\
(10)\end{array}$ & $\begin{array}{l}\text { Lu: “sister } \\
\text { es primo/" } \\
\text { (17) }\end{array}$ & $\begin{array}{l}8^{\text {a }} \text { posición } \\
\text { heteroiniciada }\end{array}$ & $\begin{array}{l}17- \\
21\end{array}$ & No & - & - \\
\hline 5 & "of & Sa: "in & $2^{\text {a }}$ posición & - & No & - & - \\
\hline
\end{tabular}

Bellaterra Journal of Teaching \& Learning Language \& Literature. 3.2 (June-July 2010): 137-171. ISSN 2013-6196. 


\begin{tabular}{|c|c|c|c|c|c|c|c|}
\hline & $\begin{array}{l}\text { bernardo" } \\
(24)\end{array}$ & $\begin{array}{l}\text { bernardol” } \\
\text { (25) }\end{array}$ & heteroiniciada & & & & \\
\hline 6 & $\begin{array}{l}\text { Ir: } \\
\text { "sha.rks" } \\
\text { (26) }\end{array}$ & $\begin{array}{l}\text { Lu: } \\
\text { "sharks〉” } \\
\text { (27) }\end{array}$ & $\begin{array}{l}2^{\mathrm{a}} \text { posición } \\
\text { heteroiniciada }\end{array}$ & $\begin{array}{l}29- \\
32, \\
34\end{array}$ & Sí & $\begin{array}{l}\text { Ir: “yes〉” } \\
\text { (35) }\end{array}$ & $\begin{array}{l}\text { Auto- } \\
\text { completada }\end{array}$ \\
\hline 7 & $\begin{array}{l}\text { Ir: "rival” } \\
\text { (37) }\end{array}$ & \begin{tabular}{l} 
Ir: "are \\
rivalry\} $\\
{\text { are }} \\
{\text { rivaly〉” }} \\
{\text { (37) }}$ & $\begin{array}{l}1^{\mathrm{a}} \text { posición } \\
\text { autoiniciada }\end{array}$ & $\begin{array}{l}37- \\
38\end{array}$ & Sí & $\begin{array}{l}\text { Lu: “que } \\
\text { son } \\
\text { rivales〉" } \\
\text { (38) }\end{array}$ & $\begin{array}{l}\text { Hetero- } \\
\text { completada }\end{array}$ \\
\hline 8 & $\begin{array}{l}\text { Ir: “n.. } \\
\text { no..” (39) }\end{array}$ & $\begin{array}{l}\text { Sa: "se } \\
\text { quieren" } \\
\text { (40) }\end{array}$ & $\begin{array}{l}2^{\mathrm{a}} \text { posición } \\
\text { heteroiniciada }\end{array}$ & $\begin{array}{l}40- \\
44\end{array}$ & Sí & $\begin{array}{l}\text { Lu: “no } \\
\text { están en } \\
\text { una } \\
\text { bandal” } \\
\text { (44) }\end{array}$ & $\begin{array}{l}\text { Hetero- } \\
\text { completada }\end{array}$ \\
\hline
\end{tabular}
\end{tabular}

Fig. 9. Reparaciones del fragmento 2

Analizaremos ahora, una por una, cada reparación. En la primera, el intento de reparación de Sa (3) en segunda posición se ve rechazado por Ir, que mantiene el mismo mensaje (4) que antes de la interrupción de Sa. Así, podemos decir que nos encontramos con la primera evidencia de una reparación sin éxito. En este caso no se trata de una reparación propiciada por un problema semántico, sino que Sa intenta acabar la oración de Ir viendo las dudas que ésta tiene (1-2) y hace una reparación de tipo morfológico (convirtiendo el determinante "two" en un pronombre).

La segunda reparación sí que es de tipo semántico: Lu no comprende el significado de "between" (4) y se lo hace saber a Ir interrumpiéndola inmediatamente (5) en una reparación también en segunda posición. Ir acaba la oración (6) sin contestar a Lu, el cual vuelve a insistir (7). Ir completa la 
reparación cambiando de código al castellano, traduciendo directamente la palabra (8).

La tercera reparación va inmediatamente después de la segunda y también es de tipo semántico: Lu no entiende qué quiere decir "teenagers” (6). Al haber habido el desarrollo de la segunda reparación entre la fuente del tercer problema y el inicio de la tercera reparación, nos encontramos con una reparación en $4^{\mathrm{a}}$ posición que se desarrolla en varios TCU: primero Ir contesta a Lu cambiando de código al castellano (10) pero lo hace en voz baja e inmediatamente retoma el discurso. Sa, que seguramente no ha oído a Ir, la vuelve a cortar y da evidencias de que no hay intersubjetividad por su parte porque no sabe cómo acabar su oración (11). Ir le ayuda (12) pero Sa sigue demostrando que no comparte el significado de "teenagers" (13). En voz baja, Ir traduce con insistencia de nuevo la palabra (14), que Sa no reconoce y, por lo tanto, le pide que la deletree (15), cosa que Ir hace (16), dando por completada la reparación.

La cuarta reparación tiene una posición muy alejada a su problema ( $8^{\mathrm{a}}$ posición) porque en medio ha habido todo el desarrollo de la reparación anterior. Lu no comprende el término “sister”, producido por Ir (10) y así se lo comunica a sus interlocutoras (17). Como las otras dos contestan al unísono (18 y 20), este solapamiento produce que a Lu no le acabe de quedar claro (19 y 21). Ir, sin embargo, continúa con su discurso sin 
más aclaraciones (22). Por lo tanto, volvemos a encontrar una reparación sin éxito.

La quinta reparación, en segunda posición, tampoco tiene éxito. Sa cree que Ir se ha equivocado de preposición (24) y se lo hace saber (25), pero Ir ignora esta interrupción y continúa con su TCU (26).

La sexta reparación tiene un desarrollo bastante extenso pero los interlocutores sí que llegan a establecer intersubjetividad. El problema vuelve a ser de tipo semántico, porque Lu no comparte con Ir la referencia "sharks" (26). Primero, repite la palabra (27) pero no especifica que no la entiende, por lo que Ir pretende continuar (28), pero su discurso se solapa con la pregunta explícita de Lu por el significado de tal palabra (29). Como Ir considera que una traducción de la palabra aquí no sería suficiente para compartir todo el contenido que comporta el término, lo explica sin cambiar de código (30). Sa hace una intervención que no resulta inteligible (31) y que dejaremos al margen del análisis. Ir parece ignorar también la intervención de Sa porque continúa con su definición de “sharks” (32), que da por satisfactoria porque pretende continuar el mensaje (33) pero se ve interrumpida nuevamente por Lu, que sí que cambia de código (34) para cerciorarse de que lo que ha entendido es correcto, cosa que Ir confirma (35), completando el desarrollo de la reparación. 
La séptima reparación que nos encontramos es la primera que autoinicia Ir en primera posición y completa otra persona. Ir tiene un problema de tipo léxico: no sabe cómo se dice "rival" en inglés y hace varias tentativas (37). Lu, inmediatamente después, le confirma a Ir que sabe lo que ella quiere decir, cambiando de código al castellano (38).

Por último, Ir vuelve a iniciar una reparación en primera posición porque tiene dudas sobre cómo acabar la oración (39). El primer intento de Sa de completar la reparación (40), en segunda posición, es rechazado por Ir (41). Lu lo intenta también pero tampoco sabe cómo acabar la oración (42). Ir reformula el mensaje pero tampoco lo consigue (43). Finalmente, Lu lo vuelve a intentar (44), primero en inglés y después en castellano, dando por completada la reparación.

En este fragmento hay muchos cambios de código, lo que demuestra nuevamente que los alumnos tienen prisa por comunicar el mensaje, por lo que buscan las soluciones más rápidas para solucionar los problemas que van surgiendo.

En cuanto a la intersubjetividad, en este fragmento encontramos que en las reparaciones iniciadas por Lu (reparaciones 2, 3 y 6), sí que hay evidencias de que Ir y $\mathrm{Lu}$ mantienen la intersubjetividad, pero no hay muestras observables que aclaren si Sa la mantiene. 


\section{Resultados}

Hemos visto que la diversidad de correspondencias entre los textos originales y el examen va en paralelo a la diversidad de tipos de interacciones en las sesiones de clase.

Sobre Porgy and Bess, vemos que la respuesta al examen es incorrecta pero la información que el alumnado recuerda coincide con el subfragmento más extenso de negociación de intersubjetividad: las nueve intervenciones que el trío dedica a negociar qué quiere decir "africans americans", aunque la pregunta buscara otra información. De la segunda idea de Lu no hay rastros en el examen ni rastros de negociación de intersubjetividad en la conversación.

En el fragmento de West Side Story, las múltiples intervenciones del trío nos hacen hablar de un grado de negociación de la intersubjetividad muy elevado. Podemos relacionar la elevada negociación con el éxito en el examen: tanto en todo este fragmento como en la primera idea del fragmento 1 se consigue construir conocimiento de ciertos contenidos. Sin embargo, hemos comprobado que la negociación de significados no era considerada por Ir, Sa y Lu como a una oportunidad de aprendizaje sino como una solución rápida para salir del paso. 
También podemos establecer un vínculo entre un tipo de interacción similar a la conversación ordinaria y la construcción de conocimientos. Los fragmentos presentan cuatro de las características de las conversaciones ordinarias: control local de la secuencia de turnos, igualdad de poder, distribución de turnos desequilibrada e intervenciones reducidas que co-construyen turnos con una sintaxis progresivamente más compleja.

En cuanto a las reparaciones, sintetizamos los datos encontrados para comprobar cómo cuajan con las conclusiones de los otros autores (cf. Figura 10):

\begin{tabular}{|l|l|l|l|}
\hline Reparación & Posición & Inicio & Éxito/Compleción \\
\hline 1.1 & $3^{\mathrm{a}}$ & Autoiniciada & Autocompletada \\
\hline 1.2 & $1^{\mathrm{a}}$ & Autoiniciada & Autocompletada \\
\hline 1.3 & $1^{\mathrm{a}}$ & Autoiniciada & Autocompletada \\
\hline 2.1 & $2^{\mathrm{a}}$ & Heteroiniciada & Sin éxito \\
\hline 2.2 & $2^{\mathrm{a}}$ & Heteroiniciada & Autocompletada \\
\hline 2.3 & $4^{\mathrm{a}}$ & Heteroiniciada & Autocompletada \\
\hline 2.4 & $8^{\mathrm{a}}$ & Heteroiniciada & Sin éxito \\
\hline 2.5 & $2^{\mathrm{a}}$ & Heteroiniciada & Sin éxito \\
\hline 2.6 & $2^{\mathrm{a}}$ & Heteroiniciada & Autocompletada \\
\hline 2.7 & $1^{\mathrm{a}}$ & Autoiniciada & Heterocompletada \\
\hline 2.8 & $2^{\mathrm{a}}$ & Heteroiniciada & Heterocompletada \\
\hline
\end{tabular}

Fig. 10. Reparaciones en los fragmentos analizados

Las reparaciones presentes en los fragmentos analizados nos muestran dos tendencias. Primero, vemos que la mayoría de reparaciones son autocompletadas, como en las conversaciones ordinarias en L2. Segundo, en cuanto a la posición, hay más 
muestras de reparaciones en segunda posición, una de las tendencias en las conversaciones en L2 en clases centradas en la lengua. Aparte de las dos apuntadas, los otros datos no nos comunican más tendencias.

\section{Conclusiones}

En la presente investigación pretendíamos encontrar si hay algún tipo de vínculo entre las características del sistema de interacciones de grupos reducidos en las aulas AICLE y el aprendizaje de contenidos.

Un primer acercamiento a los resultados de los análisis nos lleva a concluir que cuanto más se acercó la conversación en el aula AICLE a las conversaciones ordinarias, es decir, cuanta más igualdad de poder hubo en las interacciones (los interlocutores colaboraron más en la construcción de los significados del mensaje e hicieron, mediante TCU reducidas, que el turno fuera progresivamente más complejo), mejor rendimiento tuvieron en el examen $\mathrm{y}$, por lo tanto, podemos hablar de una mayor construcción de conocimientos.

Esta primera conclusión se sustenta también en lo referente a las reparaciones: un mayor número de reparaciones implicó una mayor negociación de la intersubjetividad (por lo menos en lo referente a Ir y Lu en el segundo fragmento, dado que Sa no dio evidencias explicitas de compartir la mencionada 
subjetividad), lo que conllevó una mayor construcción de conocimientos.

Creemos que un aspecto vital para proseguir con la investigación aquí comenzada sería analizar la grabación de la conversación durante el examen, porque nos permitiría seguir cómo narra el trío su proceso de aprendizaje y nos aportaría más pistas sobre cómo cada individuo ha construido dichos conocimientos. De momento, esto no nos ha sido posible.

\section{Referencias bibliográficas}

Coyle, D. (1999) The next stage? Is there a Future for the Present? The legacy of the 'communicative approach'. Francophonie, 19: 13-16.

Dalton-Puffer, C. (2007) Discourse in Content and Language Integrated Learning. Amsterdam: John Benjamins.

Markee, N. (2000) Conversation Analysis. Mahwah, N. J.: Lawrence Erlbaum.

Schwartz, J. (1980) The negotiation for meaning: repair in conversations between second language learners of English. In Discourse Analysis in Second Language Research, D. Larsen-Freeman (Ed.),. Rowley, MA: Newbury House Publishers.

Seedhouse, P. (2005) Conversation Analysis and language learning. State-of-the-Art Article. Language Teaching, 38:167-187.

Varonis, E.M. y Gass, S. (1985) Non-native / non-native conversations: A model for negotiation of meaning. Applied Linguistics, 6:71-90. 


\section{Anexo A - Secuencia didáctica}

Características generales

- Nombre: Let's musicalize in English!

- Áreas implicadas: Música e inglés.

- Nivel: 3r ESO.

- Número de alumnos: 22.

- Temporalización: Tercer trimestre.

- $\quad$ Número de sesiones: 12 sesiones de una hora.

Objetivos

- Escuchar comprensivamente música de estilos y culturas diversas, con interés, respeto y sensibilidad estética.

- Distinguir, por medio de la audición, los estilos más importantes de la historia de la música.

- Comprender los diferentes estilos de musicales del siglo XX.

- Reflexionar sobre la dimensión social de la música y comprender sus relaciones con otros ámbitos artísticos y con los medios de comunicación.

- Relacionar los compositores y sus obras con otros sucesos históricos, artísticos y sociales de la época.

- Reconocer la importancia de la música en el cine y el teatro.

- Buscar y comprender información procedente de diferentes fuentes y medios para enriquecer los conocimientos musicales propios.

- Comunicar conocimientos musicales de forma oral y escrita, con rigor y claridad.

- Investigar los aspectos musicales y extramusicales relacionados con la obra musical trabajada y expresar los resultados oralmente y por escrito.

- $\quad$ Comunicar opiniones musicales, respetando a las personas y los valores democráticos.

- $\quad$ Respetar todo hecho musical, aceptando criterios y gustos diferentes y exponiendo el criterio propio con argumentos lógicos y razonados. 
- Valorar la audición musical como fuente de placer, de enriquecimiento y crecimiento personal y del mundo.

Contenidos

- Conceptos

- Los musicales del siglo XX.

- $\quad$ Porgy and Bess (1935). George Gershwin (EEUU).

- West Side Story (1957). Leonard Bernstein (EEUU).

- $\quad$ Cats (1981). Andrew Lloyd Webber (Reino Unido).

Procedimientos

- $\quad$ Audición activa de fragmentos musicales significativos.

- Identificación auditiva de los instrumentos y los grupos instrumentales.

- Identificación de la forma y de la estructura interna de la obra musical.

- Identificación y comparación de estilos y versiones mediante la audición.

- Identificación auditiva por comparación de la tonalidad mayor y menor mediante la audición.

- Búsqueda de información en fuentes documentales en lengua inglesa.

- Identificación de los datos importantes de la época que influyen sobre los autores y las obras trabajadas.

- Organización de la información en cuadros y tablas.

- Elaboración de resúmenes y esquemas en lengua inglesa.

- Uso de la lengua inglesa oral y escrita para exponer las sensaciones y sentimientos que despierta la obra escuchada.

Actitudes

- Interés por conocer los diferentes géneros musicales y sus funciones expresivas.

- Toma de conciencia de la influencia del contexto cultural y social en la creación de la obra musical. 
- Sensibilidad estética ante las nuevas propuestas musicales, valorando los elementos creativos e innovadores que implican.

- Conciencia del valor del trabajo en grupo.

- Conciencia del valor de la comunicación en lengua extranjera.

Conexiones de las áreas de música y lengua inglesa

- Enriquecimiento de los intercambios comunicativos.

- Integración del lenguaje musical y verbal.

- Uso de la lengua inglesa oral y escrita para analizar obras musicales.

- Uso de la lengua inglesa oral y escrita para profundizar en los conocimientos musicales y sociales.

- Uso de la lengua inglesa oral y escrita para expresar sentimientos y emociones, ligados a la audición musical.

Temporalización

\begin{tabular}{|l|l|}
\hline Sesión & Contenido \\
\hline 1 & Musical films - An introduction \\
\hline 2 & Porgy and Bess (1935). George Gershwin (EEUU). \\
\hline 3 & "Summertime” \\
\hline 4 & West Side Story (1957). Leonard Bernstein (EEUU). \\
\hline 5 & "America” \\
\hline 6 & Cats (1981). Andrew Lloyd Webber (UK). \\
\hline 7 & $\begin{array}{l}\text { "Memory", "Skimbleshanks and Mungojerrie” and } \\
\text { "Rumpelteazer" }\end{array}$ \\
\hline 8 & Exam \\
\hline
\end{tabular}

Criterios de evaluación

- Comunicar de forma oral y escrita los conocimientos adquiridos, utilizando correctamente el vocabulario musical adquirido.

- Expresar opiniones musicales propias, fundamentándolas en la sensibilidad musical adquirida, utilizando correctamente el vocabulario musical adquirido. 
Instrumentos de evaluación

- Grabaciones en el aula.

- Participación activa en las actividades de todo el grupo.

- Participación activa en las actividades del trío de expertos.

- Prueba final.

- Apartado lingüístico: expresar correctamente la opinión personal sobre una audición y fundamentarla adecuadamente.

- Apartado musical: describir las características de estilo, forma, contenido y argumento de las obras trabajadas, contextualizarlas en el período histórico correspondiente y asociarlas a su autor.

\section{Anexo B - Contenidos de la SD analizados}

SESSION 2 - Porgy and Bess.

STUDENT B - PORGY AND BESS CHARACTERISTICS

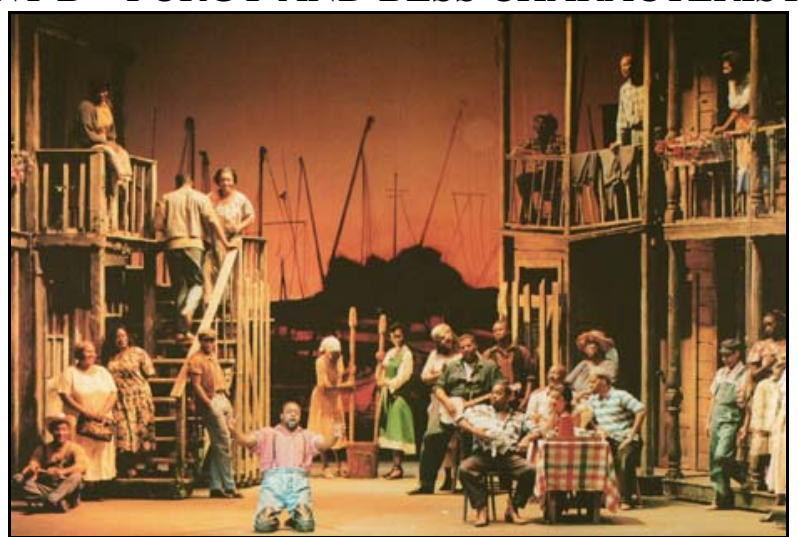

Porgy and Bess tells the story of Porgy, a disabled black man who lived in Catfish Row, a mansion in the black suburbs of Charleston (South Carolina) in the early 1920s. Porgy tries to rescue Bess from Crown, her violent and possessive lover, and Sportin' Life, the drug dealer. It is a tragic love affair. Porgy and Bess performed in New York in March 2009.

Some critics consider this opera a racist description of African Americans. Several of the members of the original cast said that they thought 
that their characters were stereotypes: African Americans lived in poverty, took drugs and solved their problems fighting.

Gershwin considered Porgy and Bess his best work. People admire Gershwin's innovative combination of European orchestral techniques with American jazz and southern black folk traditions. Gershwin composed the songs with all the folk styles he knew: jubilees, blues, praying songs, street cries, work songs, and spirituals in combination with traditional arias and recitatives.

Many people think that for many songs, Gershwin used melodies from Jewish liturgical music. The score uses leitmotifs, which relate each character with a unique musical tune. The score combines these tunes to show conflict between characters.

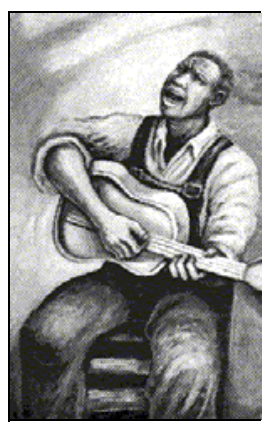

Blues

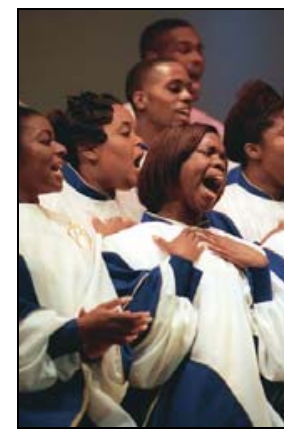

Spirituals

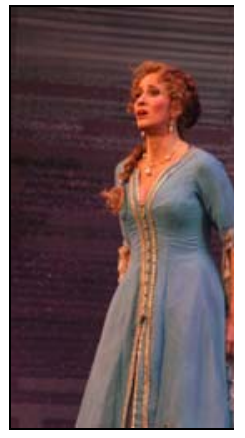

Aria

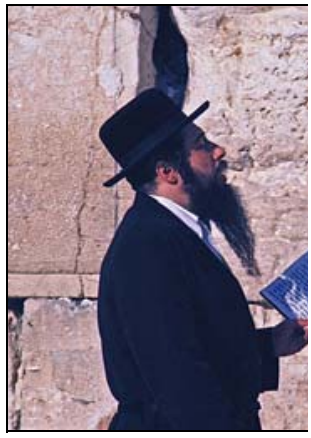

Jewish liturgical prayer 
SESSION 4 - West Side Story. STUDENT A - THE MUSICAL

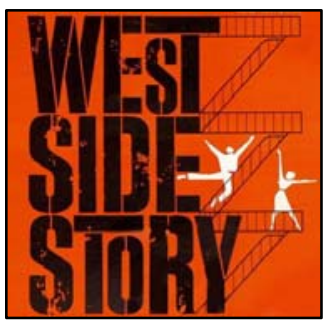

West Side Story is a musical of 1957. Leonard Bernstein composed the music and Stephen Sondheim wrote the lyrics.

West Side Story poster.

West Side Story is based on William Shakespeare's Romeo and Juliet but it is set in the West Side neighbourhood of New York in the 1950s. It explains the rivalry between two teenage gangs of different cultures: the Jets (white Americans) and the Sharks (latin Puerto Rican immigrants). The young protagonist, Tony, who belongs to the Jets, falls in love with Maria, the sister of Bernardo, the Shark's leader. The couple defies the traditional rivalry between the Jets and the Sharks, whose members don't understand their

Typical West Side buildings.

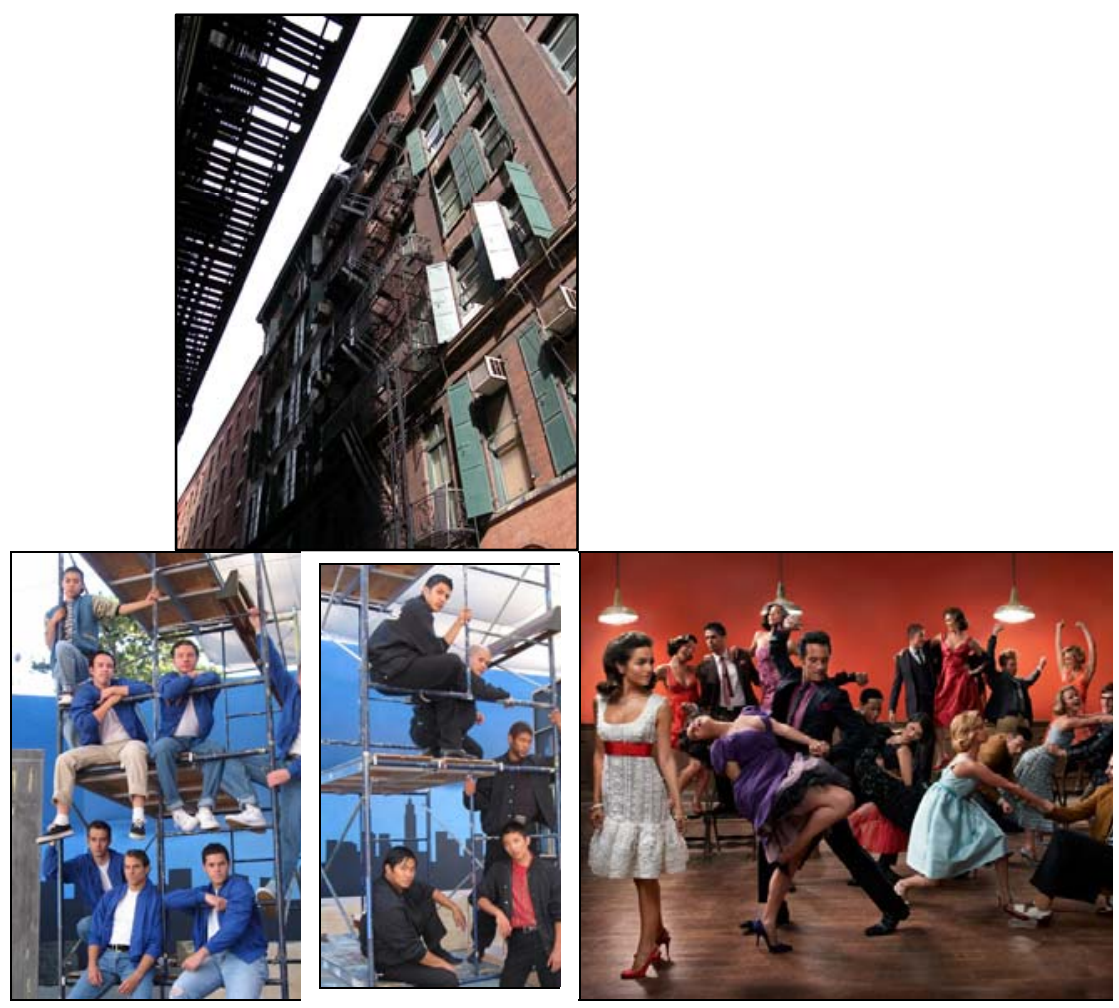

Bellaterra Journal of Teaching \& Learning Language \& Literature. 3.2 (June-July 2010): 137-171. ISSN 2013-6196. 
The Jets The Sharks When Maria and Tony met Elvis Presley and Audrey Hepburn almost played Tony and Maria roles in the 1961 film, but Elvis' manager refused the job and Hepburn got pregnant. The film won 10 Oscars.

Bernstein's score for the musical has become extremely popular. The instrumentation is incredibly large, but typical for Broadway. A total of 51 different instruments (apart from the singers' voices) are used: flutes, oboes, clarinets, saxophones, trumpets, trombones, drums, xylophones, castanets, maracas, tambourines, piano, electric guitars, Spanish guitars, mandolins, violins, cellos, etc.

A musical device in West Side Story is the tritone interval. It is dissonant (it sounds unsettled and creates musical tension). It is frequently used, especially in all the fight music. Using the tritone, Bernstein shows his musical representation of the opposing gangs. 


\section{Anexo C - Examen final del trío analizado}

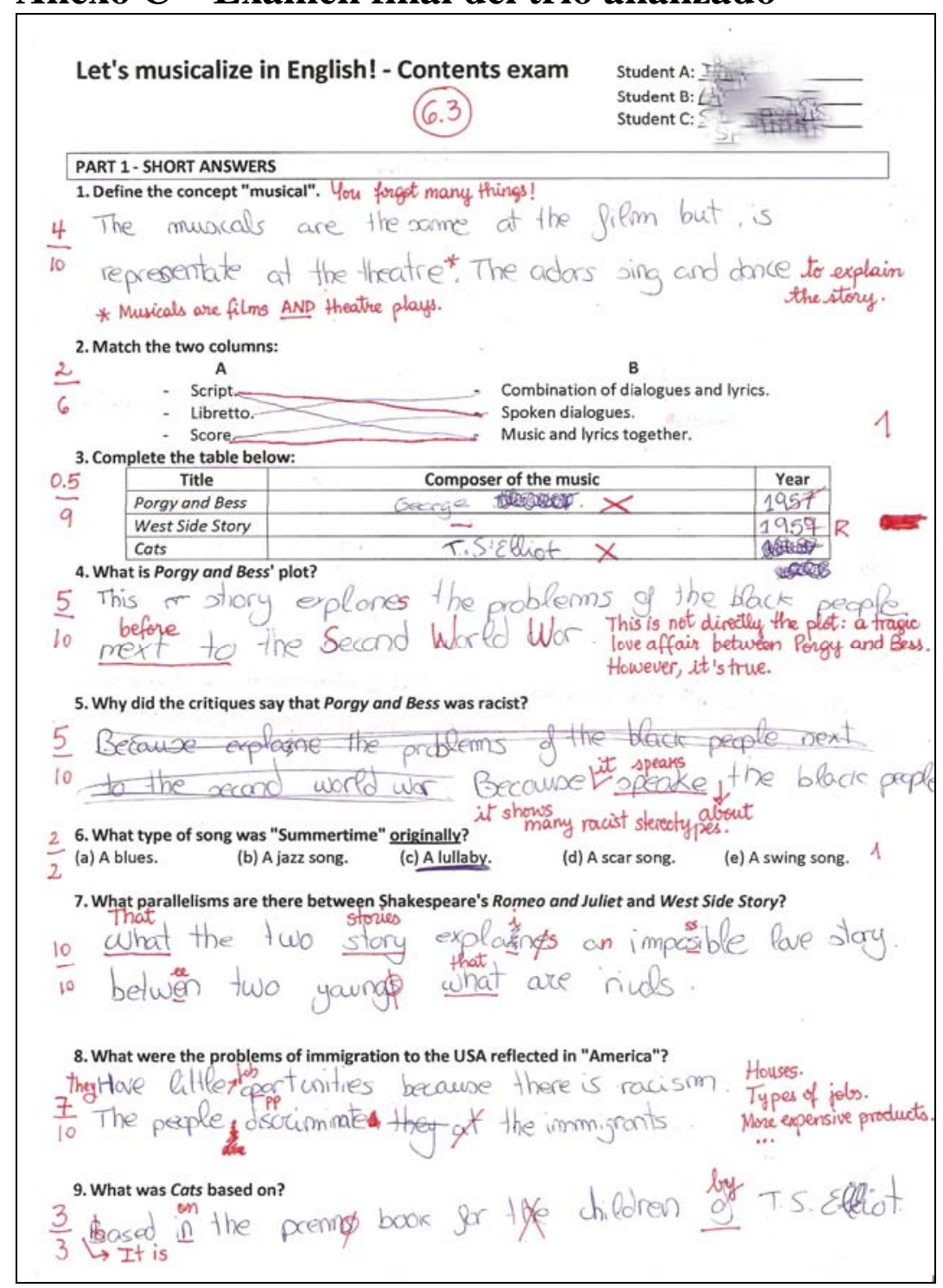

\section{Anexo D - Simbología de transcripción (del grupo CAD)}

Bellaterra Journal of Teaching \& Learning Language \& Literature. 3.2 (June-July 2010): 137-171. ISSN 2013-6196. 


\begin{tabular}{|c|c|c|c|}
\hline & Inicio de la intervención & & Pausa breve \\
\hline & Entonación descendente & $\|$ & Pausa mediana \\
\hline & Entonación ascendente & $<\mathrm{n}>$ & Pausa de $\mathrm{n}$ segundos \\
\hline & Entonación mantenida & $\ldots \ldots$ & $\begin{array}{l}\text { Alargamiento de la } \\
\text { vocal }\end{array}$ \\
\hline$=$ texto $=$ & Encabalgamientos & & Frase inacabada \\
\hline$\{(\mathrm{P})$ texto $\}$ & Volumen bajo & $\{(\mathrm{F})$ texto & Volumen alto \\
\hline $\begin{array}{l}\{(\mathrm{DC}) \text { texto } \\
\}\end{array}$ & Tempo desacelerado & $\begin{array}{l}\{(\mathrm{AC}) \text { text } \\
\text { o }\}\end{array}$ & Tempo acelerado \\
\hline$\{(\&)$ texto $\}$ & Fragmento dudoso & $\mathrm{X}$ & $\begin{array}{l}\text { Fragmento } \\
\text { incomprensible }\end{array}$ \\
\hline [+texto+] & Comentarios fonéticos & [texto] & $\begin{array}{l}\text { Comentarios de } \\
\text { transcripción }\end{array}$ \\
\hline$\{($ cas)texto & \multicolumn{3}{|c|}{ Cambio de código al castellano } \\
\hline
\end{tabular}

Referencia de la autora:

Berta de Dios Garcia es licenciada en Traducción e Interpretación por la Universitat Pompeu Fabra y tiene el Máster Oficial en Investigación en Didáctica de la Lengua y de la Literatura por la Universitat Autònoma de Barcelona. Ha trabajado en varios institutos de secundaria y escuelas oficiales de idiomas del Vallès Occidental y Oriental.

\section{Email: bertadedios@gmail.com}

1 "Las reparaciones en segunda posición tienen lugar en el turno [TCU] siguiente a la fuente problemática y son iniciadas por otro [interlocutor] (...). No solamente inician una secuencia de reparación sino que también contienen una potencial compleción (...) que facilita la resolución per parte del primer hablante en el siguiente (tercer) turno [TCU]. Aunque ésta es la trayectoria que suelen seguir las reparaciones en segunda posición, puede darse más reparación en varios turnos [TCU] subsiguientes, resultando en una autoreparación o una reparación per parte de otra persona” (Markee 2000:102).

2 "Las reparaciones en tercera posición tienen lugar en el tercer turno [TCU] de una secuencia de reparación, en las siguientes circunstancias: el hablante A produce un primer turno [TCU] aparentemente adecuado (T1), que el hablante $\mathrm{B}$, por tanto, trata como tal y al que contesta en el T2 (...). A pesar de ello, en el T3, el hablante A hace entender a B que su comprensión del T1 era errónea, iniciando un trabajo que repara esta comprensión equivocada.” (Markee 2000:102). 